\title{
High national and sub-national coverage of iodised salt in India: evidence from the first National lodine and Salt Intake Survey (NISI) 2014-2015
}

\author{
Chandrakant S Pandav ${ }^{1}$, Kapil Yadav ${ }^{2, *}$, Harshal R Salve ${ }^{2}$, Rakesh Kumar ${ }^{2}$, Akhil D Goel ${ }^{3}$ \\ and Arijit Chakrabarty ${ }^{4}$ \\ ${ }^{1}$ Indian Coalition for Control of lodine Deficiency Disorders (ICCIDD), New Delhi, India: ${ }^{2}$ Centre for Community \\ Medicine, All India Institute of Medical Sciences (AlIMS), Ansari Nagar, New Delhi - 1 10023, India: ${ }^{3}$ Department \\ of Community Medicine and Family Medicine, All India Institute of Medical Sciences (AllMS), Jodhpur, India: \\ ${ }^{4}$ Bestseller Project, Global Alliance for Improved Nutrition (GAIN), New Delhi, India
}

Submitted 7 August 2017: Final revision received 3 August 2018: Accepted 7 August 2018: First published online 10 September 2018

\begin{abstract}
Objective: The National Iodine and Salt Intake Survey (NISI) 2014-2015 was undertaken to estimate household iodised salt coverage at national and subnational levels in India.

Design: Cross-sectional survey with multistage stratified random sampling.

Setting: India was divided into six geographic zones (South, West, Central, North, East and North-East) and each zone was further stratified into rural and urban areas to yield twelve distinct survey strata.

Subjects: The target respondent from each household was selected as per predefined priority; wife of the household head, followed by women of reproductive age, followed by any adult available during the visit.

Results: Households ( $n$ 5717) were surveyed and salt samples ( $n$ 5682) were analysed. Household coverage of iodised salt (iodine $\geq 5 \mathrm{ppm}$ ) was 91.7 (95\% CI $91 \cdot 0,92 \cdot 7) \%$. Adequately iodised salt (iodine $\geq 15 \mathrm{ppm}$ ) was consumed in $77 \cdot 5$ (95\% CI 76.4, 78.6) \% of households. Significant differences in coverage were seen across six geographic regions, with North and North-East zones on the verge of achieving the universal salt iodisation target of $>90 \%$ coverage. Coverage of households with adequately iodised salt (adjusted OR; 95\% CI) was significantly less in rural households $(0.55 ; 0.47,0.64)$, lower/backward castes $(0.84 ; 0.72$, $0 \cdot 98)$, deprived households $(0.72 ; 0.61,0.85)$ as assessed by multidimensional poverty index, households with non-diverse diet $(0 \cdot 73 ; 0 \cdot 62,0 \cdot 86)$ and households using non-packaged salt $(0 \cdot 48 ; 0 \cdot 39,0.59)$ and non-refined salt $(0 \cdot 17 ; 0 \cdot 15,0 \cdot 20)$. Conclusions: India is within striking reach of achieving universal salt iodisation. However, significant differentials by rural/urban, zonal and socio-economic indicators exist, warranting accelerated efforts and targeted interventions for highrisk groups.
\end{abstract}

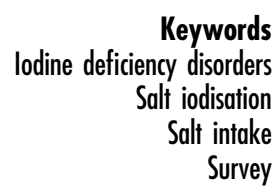

Globally, 1.8 billion people are at risk of iodine deficiency disorders (IDD), significantly affecting human development potential at the global and national levels ${ }^{(1)}$. Iodine deficiency is the single most important cause of preventable brain damage globally. Children born in iodine-deficient areas have higher rates of neonatal hypothyroidism ${ }^{(2)}$, underweight $^{(3)}$, impaired growth ${ }^{(4)}$, learning disabilities ${ }^{(5)}$, mental retardation, psychomotor defects, hearing and speech impairments, and low intelligence quotient ${ }^{(6)}$. Antenatal iodine deficiency has been linked with adverse birth outcomes including abortion, stillbirth and neonatal death. The association of inadequate iodised salt intake with low urinary iodine concentration has been well established in several studies from across the world. It has been shown that even mild to moderate deficiency of iodine intake can lead to learning disabilities and inadequate mental and physical development ${ }^{(5,7)}$.

In India the entire population is at risk of IDD because, due to glaciation, deforestation and repeated floods, the soil of the subcontinent and consequently the food derived from it is iodine deficient. Surveys conducted by central and state health directorates, the Indian Council of 
Medical Research and medical institutes since the 1950s have clearly demonstrated that IDD is a public health problem in all states and union territories in India. Statelevel IDD surveys carried out in seven states (Kerala, Tamil Nadu, Orissa, Rajasthan, Bihar, Goa and Jharkhand) from 2000 to 2006 also showed endemicity of IDD in all surveyed states ${ }^{(8)}$.

Universal salt iodisation (USI) has been globally recognised as the primary strategy for the prevention and control of IDD ${ }^{(9,10)}$. India was one of the first countries in the world to initiate a salt iodisation programme in the year $1962^{(11)}$. The key objectives of the National Iodine Deficiency Disorders Control Programme (NIDDCP) are: to assess the magnitude of IDD and the supply of iodised salt in place of common salt; to resurvey after every 5 years to assess the extent of IDD and the impact of iodised salt; laboratory monitoring of iodised salt and urinary iodine excretion; and health education and advocacy ${ }^{(12)}$. The NIDDCP has been a public health success story in India with household coverage of adequately iodised salt showing a consistent increase; from $<5 \%$ in 1985 to $51 \%$ in the National Family Health Survey-3 (NFHS-3; 200506) ${ }^{(13)}$ to $71 \%$ in the Coverage Evaluation Survey (CES; $2009)^{(14)}$ to $93 \%$ in the National Family Health Survey-4 (NFHS-4; 2015-16) ${ }^{(15)}$. All these national salt coverage estimates just mentioned are based on rapid semiquantitative salt testing kits (STK) for assessing the iodine content of household salt, which are however inferior to the laboratory-based iodometric titration method $^{(16)}$. Given the maturity of the national programme and evidence of its success, it was considered essential to generate quantitative, representative and reliable estimates of iodised salt coverage at national and sub-national levels in India. Also, as per the resolution of the World Health Assembly, all member countries are required to report progress on IDD status to the Assembly every 3 years ${ }^{(17)}$. The current national survey provided information to report IDD progress to the World Health Assembly. The National Iodine and Salt Intake Survey (NISI) 2014-2015 was undertaken to estimate household iodised salt coverage at national and regional levels (six geographical zones) in India.

\section{Methods}

A national-level house-to-house survey was conducted from September 2014 to August 2015. In order to ensure representative sampling, India was divided into six geographic zones (South, West, Central, North, East and North-East) as per the guidelines of the Inter-State Council Secretariat $^{(18)}$ (Fig. 1). Each zone was further stratified into rural and urban areas to yield twelve distinct survey strata.

Sample size estimation was based on assumption of coverage of adequately iodised salt at stratum level of $50 \%$, permissible margin of error of $8 \%$ at stratum level (reducing to $<3 \%$ in the national sample), response rate of $77 \%$ and design effect of $2 \cdot 5$. The required sample size was 490 households per stratum and a total sample size of 5880 households at national level at 95\% confidence level. To reduce the design effect while retaining cost-effectiveness, we chose forty-two primary sampling units (PSU) in each stratum and twelve households in each PSU. Thus, the final target sample size was 504 households per stratum and 6048 households nationally.

A multistage stratified random sampling strategy was used: the first stage was selection of PSU and the next stage was selection of households (Fig. 2). PSU were selected using population probability sampling, from a list of villages (PSU in rural areas) and census enumeration (a)

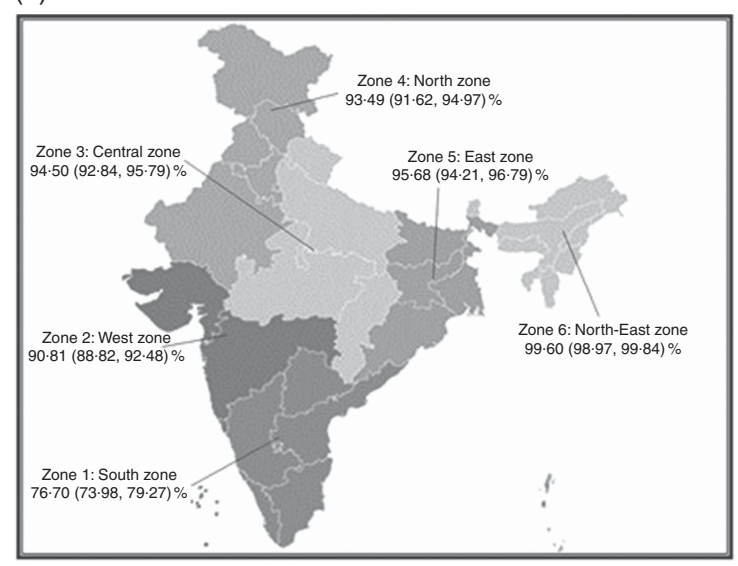

(b)

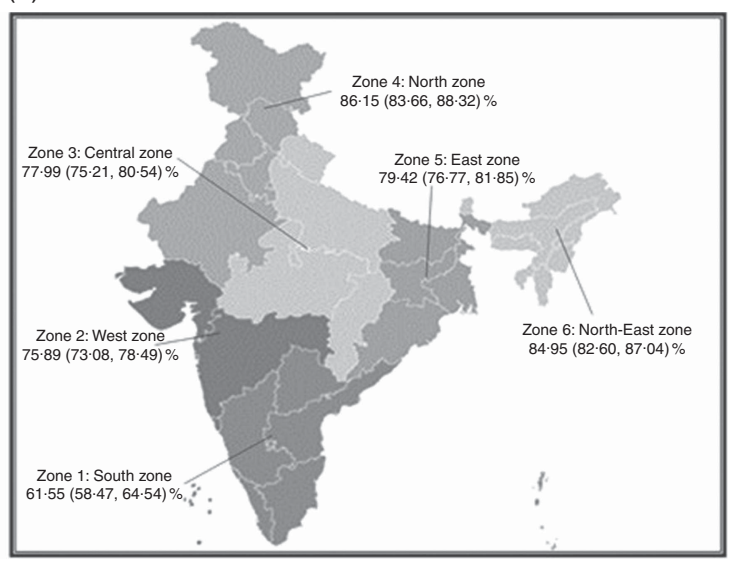

Fig. 1 Coverage of (a) iodised salt (iodine $\geq 5 \mathrm{ppm}$ ) and (b) adequately iodised salt (iodine $\geq 15 \mathrm{ppm}$ ) in various geographical zones of India; first National lodine and Salt Intake (NISI) Survey 2014-2015. Values given are coverage percentage (95\% confidence interval). South zone (zone 1)=Andhra Pradesh, Karnataka, Kerala, Tamil Nadu, Telangana; West zone (zone 2)=Gujarat, Maharashtra; Central zone (zone 3) = Uttarakhand, Uttar Pradesh, Chhattisgarh, Madhya Pradesh; North zone (zone 4) = Jammu \& Kashmir, Himachal Pradesh, Punjab, Haryana, Delhi, Rajasthan; East zone (zone 5)=Bihar, West Bengal, Jharkhand, Odisha; North-East zone (zone 6) = Arunachal Pradesh, Nagaland, Manipur, Mizoram, Tripura, Meghalaya, Assam 

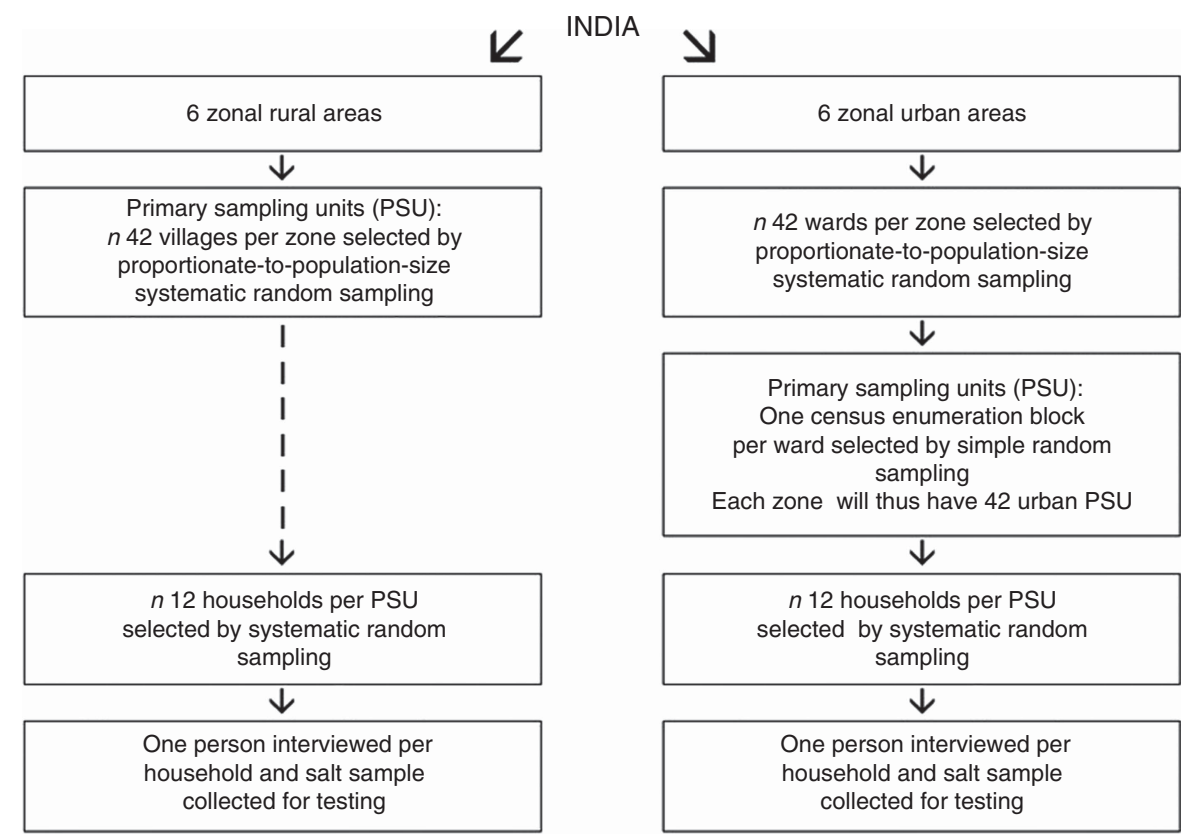

Fig. 2 Flowchart of sampling methodology of the first National lodine and Salt Intake Survey (NISI) 2014-2015

blocks (PSU in urban areas) obtained from the Registrar General of India Office. In each zone, forty-two rural PSU, defined as a village with $\leq 250$ households approximately, were selected by the proportionate-to-population-size systematic random sampling method ${ }^{(19)}$. Larger villages were divided into smaller segments of approximately 250 households to constitute separate PSU. Similarly, in urban strata in each zone, forty-two wards were first selected using proportionate-to-population-size systematic random sampling and then one census enumeration block was selected randomly from the selected ward to constitute the urban PSU. The list of rural villages and urban census enumeration blocks, and their corresponding populations, was obtained from the Census 2011.

At PSU level, after an exhaustive house-listing exercise to define the sampling frame, twelve households were selected by systematic random sampling. The target respondent from each household was selected as per predefined priority: wife of the household head, followed by women of reproductive age, followed by any adult available during the visit. No replacement policy was followed in case of non-eligible or locked households.

\section{Study tools}

A structured pre-tested interview schedule was administered to selected respondents after obtaining written informed consent. The consent form was read aloud for illiterate respondents in the presence of a literate witness and verbal consent obtained. The survey questionnaire was developed using inputs from similar fortification coverage surveys carried out in India and globally. The interview schedule included information about sociodemographic characteristics, type of salt used in the household (refined, washed, crystal or 'phoda'; packaged or loose salt), dietary diversity, Multidimensional Poverty Index (MPI) ${ }^{(20)}$, household food security ${ }^{(21)}$, and knowledge, attitudes and practices about use of iodised salt.

The MPI is a weighted index of household vulnerability to poverty, developed by the Oxford Poverty and Human Development Initiative for the United Nations Development Programme's flagship Human Development Report in $2010^{(20)}$. It includes the dimensions of education (school attainment and attendance), health (household hunger and mortality among children aged $<5$ years) and living standards (drinking-water, electricity, sanitation, type of floor, cooking fuel and assets). The MPI score ranges from 0 to 1 , and households with a score of $\geq 1 / 3$ were considered deprived $^{(22)}$.

Dietary diversity was ascertained using food intake data collected by the $24 \mathrm{~h}$ recall method, as has been used in National Nutritional Surveys ${ }^{(23)}$. Overall dietary diversity of the respondent was computed from a listing of nine food types and questions coded as $1=$ 'the respondent has eaten the food type' and $0=$ 'the respondent has not eaten the food type' (range: 0-9). Based on their food diversity score, respondents were classified into two categories: those with diverse diet (score of $\geq 4$ ) and those with nondiverse diet $(\text { score of }<4)^{(24)}$.

The Household Food Insecurity Access Scale was used to assess household anxiety about food security and indications that food was of insufficient quality or quantity $^{(21)}$. This scale measures availability and access to the required food at the household level during the past $30 \mathrm{~d}$ from the date of the survey using a set of nine questions. The households were classified into four categories based 
on the combination of responses to the nine questions, as per Coates et al.: (i) food secure, i.e. household experiences no food insecurity conditions or just experiences worry, but rarely; (ii) mildly food insecure, i.e. household worries about not having enough food sometimes or often, and/or is unable to eat preferred foods, and/or eats a more monotonous diet than desired, and/or eats some foods considered undesirable, but only rarely; (iii) moderately food insecure, i.e. household eats a monotonous diet or undesirable foods sometimes or often, and/or has started to cut back on quantity by reducing the size of meals or number of meals, rarely or sometimes; and (iv) severely food insecure, i.e. household cuts back on meal size or number of meals often, and/or experiences any of the three most severe conditions (running out of food, going to bed hungry, or going a whole day and night without eating) even as infrequently as rarely ${ }^{(21)}$.

The questionnaire was pre-tested to check the flow of questions, respondents' understandings of questions, skips and filters. The final questionnaire was then translated into ten local languages, i.e. Hindi, Gujarati, Tamil, Telugu, Kannada, Malayalam, Bengali, Marathi, Odiya and Assamese. The questionnaire was used in a bilingual format. Translated questionnaires were back-translated by independent persons to establish consistency in translation.

Informed written consent was taken from the literate participants of the survey. For illiterate respondents, the consent form was read aloud in the presence of a literate witness and verbal consent of the respondent duly signed by the witness was obtained. Ethical clearance for the survey was obtained from the Institutes Ethical Committee of the All India Institute of Medical Sciences, New Delhi.

\section{Salt sample analysis}

A sample of the salt used for cooking the meal in the household on the preceding night was collected. Approximately $50 \mathrm{~g}$ of salt was collected and packed in a self-locking zip-lock polyethylene bag. The collected salt samples were transported in a moisture-free container to the Salt Iodine Testing laboratory at the All India Institute of Medical Sciences, New Delhi, within one week of collection. Salt samples were analysed for iodine content at the laboratory of the Indian Coalition for Control of Iodine Deficiency Disorders/Iodine Global Network located at the All India Institute of Medical Sciences, New Delhi, by iodometric titration, the gold standard method for estimating iodine content in the salt ${ }^{(19)}$.

\section{Quality assurance}

Household interviews were conducted using the computer-assisted personal interview method and the software application included logical checks (validation of inputs and skips) at the time of data input during the interview itself. Fieldworkers collecting the data during the survey were trained in study questionnaire administration and salt sample collection by investigators of the survey. Faculty members (public health experts) from state medical colleges conducted external supervision in $10 \%$ of PSU after completion of the survey. External supervisors administered the survey tool and collected the salt sample from the same households as those selected in the main survey. Iodometric titration was carried out as per the standard guidelines. The iodine testing laboratory observed both internal and external quality control mechanisms ${ }^{(12)}$.

\section{Statistical analysis}

Data were entered in a spreadsheet (Microsoft ${ }^{\circledR}$ Excel for Windows 2010 edition) and analyses were done using the statistical software package IBM SPSS Statistics version 22. The Complex Samples Module in SPSS, which accounts for the design effect, was used to calculate percentages and 95\% CI of population estimates for the weighted data. The Complex Samples Analysis plan file was developed to include the strata, the PSU, the probability of selection for the PSU, the probability of selection for a household within the PSU and the weights adjusted for non-response. Weight development in the survey was done taking into account the sampling design followed and the allocation of samples made at various stages of sampling. By considering the zonal and national samples achieved, normalisation was done on the urban/rural weighted values to arrive at zonal and national weights. Distribution of household iodine salt content across three categories (nil iodine, 0-4.9 ppm; inadequate iodine, 5-14.9 ppm; adequate iodine, $\geq 15 \mathrm{ppm}$ ) was studied in relation to selected demographic (place of residence, MPI, food insecurity, etc.) and other explanatory variables (type of salt, dietary diversity). Multivariate logistic regression analysis with household salt iodine content (dichotomised as salt with no or inadequate iodine $v$. salt with adequate iodine) as dependent variable was done using selected sociodemographic and salt category variables as independent variables (place of residence, education status, caste, socio-economic status, food insecurity, dietary diversity, refined/non-refined salt, packaged/non-packaged salt). The variables to be included in multivariate analysis were determined based on the results of bivariate analyses as well as on an a priori short listing of key variables likely to affect salt iodisation levels.

\section{Results}

Overall, 5717 household interviews were conducted and 5682 salt samples were collected against a target sample size of 6048 (response rate: 94.5 and $93.9 \%$, respectively). Overall, $91.3 \%$ of the survey respondents were female, with more than four-fifths of them being of reproductive age and two-thirds from lower/backward classes. Overall, 
$73.2 \%$ of respondents had received formal education, with respondents in urban areas being more educated than respondents in rural areas. Of the households, $24.9 \%$ suffered deprivation (MPI score of $\geq 1 / 3$ ) and $18.4 \%$ experienced moderate to severe food insecurity (Table 1 ).

At the national level, the household coverage of iodised salt (iodine $\geq 5 \mathrm{ppm}$ ) was 91.7 (95\% CI 91.0, 92.4)\%, with 77.5 (95\% CI 76.4, 78.6)\% of households consuming adequately iodised salt (iodine $\geq 15$ ppm; Fig. 3). Inadequately iodised salt (iodine $=5-14.9 \mathrm{ppm}$ ) was used by $14.3 \%$ of households, and $8.3 \%$ of households were using non-iodised salt (iodine $<5$ ppm; Table 2).

At sub-national level, the North and North-East zones were doing significantly better than the national level and had nearly achieved USI (>90\% coverage of adequately iodised salt). At national level, the coverage of adequately iodised salt in rural regions lagged behind the urban regions by 16.8 percentage points (69.1 v. $85.9 \%$; Table 2 ). Similar findings were observed at zonal estimates (see online supplementary material, Supplemental Table 1). Urban regions in Central, North and North-East zones had already achieved USI.
Deprived households (MPI score $\geq 1 / 3$ ) had lower coverage of adequately iodised salt (70.2\%; $95 \%$ CI $67 \cdot 8$, $72.6 \%)$ compared with non-deprived households (MPI score $<1 / 3 ; 70.9 \%$; $95 \%$ CI 78.7, 81.1\%). Also, percentage use of non-iodised salt was higher among deprived households (9.5\%; 95\% CI 7.3, 11.9\%) compared with non-deprived households (7.7\%; 95\% CI 6.4, 8.9\%; Table 3). Similar findings were reported at zonal level (see online supplementary material, Supplemental Table 1).

Higher coverage of use of adequately iodised salt (77.9\%; 95\% CI 76.7, 79.1\%) was observed among foodsecure households compared with food-insecure households (76.4\%; $95 \% 74.2,78.5 \%$; Table 3 ). Similar findings were reported at zonal level (see online supplementary material, Supplemental Table 1).

Overall, $67 \%$ of households were using refined type of salt, with 33\% still consuming non-refined salt. The different types of non-refined salt consumed were washed salt $(23.4 \%)$, crystal salt (small granules; $7 \cdot 3 \%$ ) and 'phoda' (large granules; $2 \cdot 3 \%$ ). The use of refined iodised salt was higher in urban areas than rural areas, with nearly half of households in rural areas still using non-refined salt

Table 1 Sociodemographic characteristics of the surveyed population in India; first National lodine and Salt Intake (NISI) Survey 2014-2015*

\begin{tabular}{|c|c|c|c|c|c|c|}
\hline & \multicolumn{2}{|c|}{ Urban ( $n$ 2852) } & \multicolumn{2}{|c|}{ Rural (n 2865) } & \multicolumn{2}{|c|}{ Total $(n$ 5717) } \\
\hline & $n$ & $\%$ & $n$ & $\%$ & $n$ & $\%$ \\
\hline \multicolumn{7}{|l|}{ Age of respondent (years) } \\
\hline Mean & \multirow{2}{*}{\multicolumn{2}{|c|}{$\begin{array}{l}36.8 \\
11.1\end{array}$}} & \multirow{2}{*}{\multicolumn{2}{|c|}{$\begin{array}{l}36 \cdot 2 \\
11.6\end{array}$}} & \multicolumn{2}{|c|}{$36 \cdot 5$} \\
\hline SD & & & & & \multicolumn{2}{|c|}{11.4} \\
\hline \multicolumn{3}{|l|}{ Gender of respondent } & & & \\
\hline Male & 251 & $8 \cdot 8$ & 249 & $8 \cdot 7$ & 500 & 8.7 \\
\hline Female & 2601 & $91 \cdot 2$ & 2616 & $91 \cdot 3$ & 5217 & $91 \cdot 3$ \\
\hline \multicolumn{7}{|l|}{ Size of household } \\
\hline Mean & \multicolumn{2}{|c|}{4.7} & \multicolumn{2}{|c|}{$5 \cdot 1$} & \multicolumn{2}{|c|}{4.9} \\
\hline SD & \multicolumn{2}{|c|}{$2 \cdot 1$} & \multicolumn{2}{|c|}{$2 \cdot 2$} & \multicolumn{2}{|c|}{$2 \cdot 1$} \\
\hline \multicolumn{7}{|l|}{ Education } \\
\hline Illiterate & 569 & $20 \cdot 0$ & 964 & $33 \cdot 6$ & 1533 & $26 \cdot 8$ \\
\hline Literate & 2283 & $80 \cdot 0$ & 1901 & $66 \cdot 4$ & 4184 & $73 \cdot 2$ \\
\hline \multicolumn{7}{|l|}{ Religion } \\
\hline Hindu & 2202 & $77 \cdot 2$ & 2238 & $78 \cdot 1$ & 4440 & $77 \cdot 7$ \\
\hline Muslim & 451 & $15 \cdot 8$ & 411 & $14 \cdot 3$ & 862 & $15 \cdot 1$ \\
\hline Christian & 135 & 4.7 & 124 & $4 \cdot 3$ & 259 & 4.5 \\
\hline Others & 64 & $2 \cdot 2$ & 92 & $3 \cdot 2$ & 156 & $2 \cdot 7$ \\
\hline \multicolumn{7}{|l|}{ Caste } \\
\hline General caste & 1070 & $37 \cdot 8$ & 825 & $29 \cdot 0$ & 1895 & 33.4 \\
\hline Other backward/lower class & 943 & 33.4 & 991 & $34 \cdot 8$ & 1934 & $34 \cdot 1$ \\
\hline Scheduled tribe & 231 & 8.2 & 360 & $12 \cdot 6$ & 591 & $10 \cdot 4$ \\
\hline Scheduled caste & 583 & $20 \cdot 6$ & 671 & $23 \cdot 6$ & 1254 & $22 \cdot 1$ \\
\hline \multicolumn{7}{|l|}{ Multidimensional Poverty Index (MPI) } \\
\hline Deprived (MPI score $\geq 1 / 3$ ) & 452 & $15 \cdot 9$ & 970 & 33.9 & 1422 & 24.9 \\
\hline Not deprived (MPI score $<1 / 3$ ) & 2398 & $84 \cdot 1$ & 1891 & $66 \cdot 1$ & 4289 & $75 \cdot 1$ \\
\hline \multicolumn{7}{|l|}{ Household food insecurity } \\
\hline Food secure & 2125 & 74.5 & 2043 & $71 \cdot 3$ & 4168 & $72 \cdot 9$ \\
\hline Mild food insecure & 245 & $8 \cdot 6$ & 255 & 8.9 & 500 & 8.7 \\
\hline Moderately food insecure & 135 & 4.7 & 155 & 5.4 & 290 & $5 \cdot 1$ \\
\hline Severely food insecure & 347 & $12 \cdot 2$ & 412 & 14.4 & 759 & $13 \cdot 3$ \\
\hline \multicolumn{7}{|l|}{ Type of diet } \\
\hline Diverse diet & 1049 & $36 \cdot 8$ & 849 & $29 \cdot 6$ & 1898 & $33 \cdot 2$ \\
\hline Not diverse diet & 1803 & $63 \cdot 2$ & 2016 & $70 \cdot 4$ & 3819 & $66 \cdot 8$ \\
\hline
\end{tabular}

${ }^{*}$ Percentages represent column-wise percentages (unless specified otherwise). 


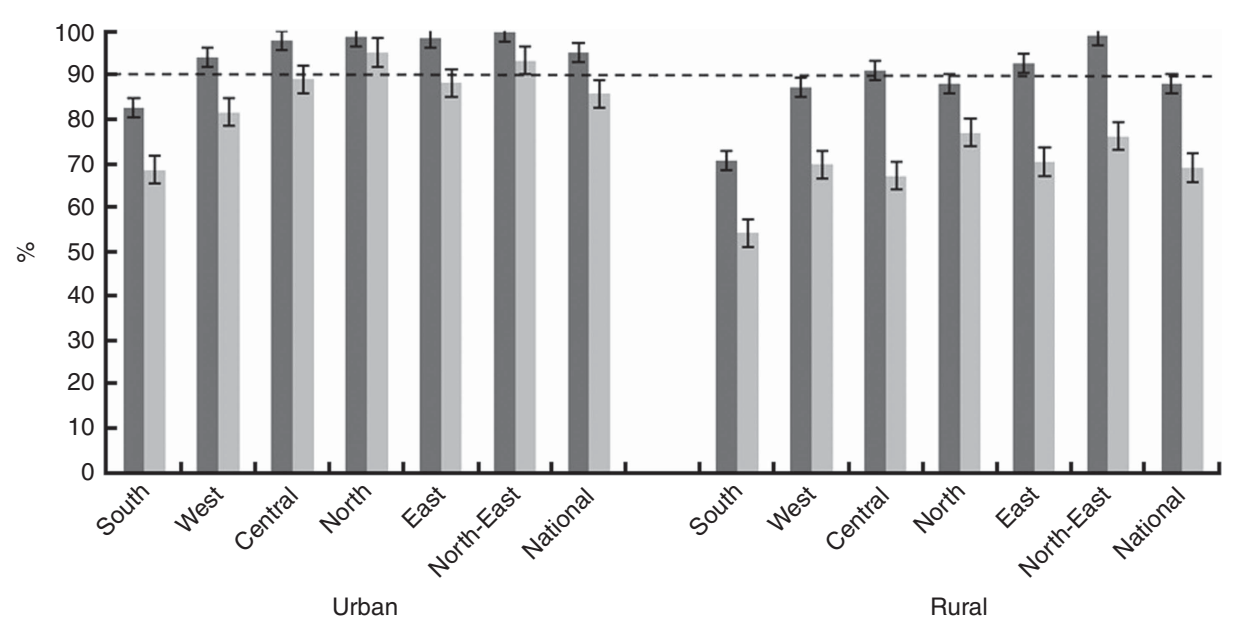

Fig. 3 National and sub-national coverage of iodised salt (iodine $\geq 5$ ppm; $\square$ ) and adequately iodised salt (iodine $\geq 15$ ppm; $\square$ ) in urban and rural strata in India; first National lodine and Salt Intake (NISI) Survey 2014-2015. Values are coverage percentages with their $95 \%$ confidence intervals represented by vertical bars; - - - represents the universal salt iodisation target of $90 \%$ coverage

Table 2 Usage patterns and iodine content of household salt in India; first National lodine and Salt Intake (NISI) Survey 2014-2015*

\begin{tabular}{|c|c|c|c|c|c|c|}
\hline & \multicolumn{2}{|c|}{ Urban (n 2852) } & \multicolumn{2}{|c|}{ Rural ( $n$ 2865) } & \multicolumn{2}{|c|}{ Total $(n$ 5717) } \\
\hline & $n$ & $\%$ & $n$ & $\%$ & $n$ & $\%$ \\
\hline \multicolumn{7}{|l|}{ Salt usage patterns } \\
\hline Use of packaged salt & 2649 & 92.9 & 2474 & $86 \cdot 4$ & 5123 & $89 \cdot 6$ \\
\hline \multicolumn{7}{|l|}{ Type of salt used } \\
\hline Refined & 2181 & $77 \cdot 1$ & 1603 & $56 \cdot 8$ & 3784 & $67 \cdot 0$ \\
\hline Washed & 480 & $17 \cdot 0$ & 841 & 29.8 & 1321 & 23.4 \\
\hline Crystal & 152 & 5.4 & 262 & $9 \cdot 3$ & 414 & $7 \cdot 3$ \\
\hline 'Phoda' & 14 & 0.5 & 114 & 4.0 & 128 & $2 \cdot 3$ \\
\hline lodine content of salt samples using iodometric titration & \multicolumn{2}{|c|}{ (n 2838) } & \multicolumn{2}{|c|}{$(n 2844)$} & \multicolumn{2}{|c|}{$(n 5682)$} \\
\hline Non-iodised (<5 ppm) & 134 & 4.7 & 335 & 11.8 & 469 & 8.3 \\
\hline Inadequately iodised (5-14.9 ppm) & 266 & $9 \cdot 4$ & 545 & $19 \cdot 2$ & 811 & $14 \cdot 3$ \\
\hline Adequately iodised salt ( $\geq 15 \mathrm{ppm})$ & 2438 & $85 \cdot 9$ & 1964 & $69 \cdot 1$ & 4402 & $77 \cdot 5$ \\
\hline \multicolumn{7}{|l|}{ Adequately iodised salt categorised } \\
\hline $15-29.9$ ppm & 1202 & $42 \cdot 4$ & 1132 & 39.8 & 2334 & $41 \cdot 1$ \\
\hline$\geq 30 \mathrm{ppm}$ & 1236 & 43.6 & 832 & $29 \cdot 3$ & 2068 & $36 \cdot 4$ \\
\hline lodised salt ( $\geq 5 \mathrm{ppm})$ & 2704 & $95 \cdot 3$ & 2509 & 88.2 & 5213 & 91.7 \\
\hline
\end{tabular}

${ }^{*}$ Percentages represent column-wise percentages (unless specified otherwise).

(Table 2). The adequately iodised salt coverage was low in households using washed $(65.8 \%$ coverage), crystal $(22.7 \%)$ and 'phoda' salt (28.9\%) compared with households using refined salt (89.4\%; Table 3 ). More than half households (57.2\%) using crystal salt had less than 5 ppm iodine in their salt. The coverage of adequately iodised salt was significantly higher in households using packaged salt (80.0\%) compared with households using non-packaged salt $(55 \cdot 2 \%)$.

In multivariate analysis, consumption of adequately iodised salt was significantly less in rural households ( $\mathrm{AOR}=0.55 ; 95 \% \mathrm{CI} 0.47,0.64)$, lower/backward castes (AOR $=0.84 ; 95 \%$ CI $0.72,0.98)$, deprived households $(\mathrm{AOR}=0.72 ; 95 \% \mathrm{CI} 0 \cdot 61,0.85)$, households with nondiverse diet $(\mathrm{AOR}=0.73 ; 95 \% \mathrm{CI} 0.62,0.86)$, households using non-packaged salt $(\mathrm{AOR}=0.48 ; 95 \%$ CI $0.39,0.59)$ and households using non-refined salt $(\mathrm{AOR}=0 \cdot 17 ; 95 \%$ CI 0.15, 0.20; Table 4).

\section{Discussion}

The present findings from the NISI 2014-2015, the first national-level IDD survey in India, highlight the notable progress made towards USI in India. There has been a sustained increase in the coverage of adequately iodised salt in India over the last decade compared with earlier available surveys, i.e. NFHS-3 (2005-06) and CES $(2009)^{(13,14)}$. India is within striking reach of achieving the USI target of greater than $90 \%$ adequately iodised salt coverage. The USI target has already been achieved in urban areas of the Central, North and North-East zones; 
Table 3 Household salt iodine content categories (quantitative) by selected characteristics in India; first National lodine and Salt Intake (NISI) Survey 2014-2015*

\begin{tabular}{|c|c|c|c|c|c|c|c|c|c|c|c|}
\hline & \multirow{3}{*}{$\frac{\text { Total }}{n}$} & \multirow{2}{*}{\multicolumn{2}{|c|}{$\begin{array}{l}\text { Non-iodised } \\
(<5 \mathrm{ppm})\end{array}$}} & \multirow{2}{*}{\multicolumn{2}{|c|}{$\begin{array}{l}\text { Inadequately iodised } \\
(5-14.9 \mathrm{ppm})\end{array}$}} & \multirow{2}{*}{\multicolumn{2}{|c|}{$\begin{array}{l}\text { Adequately iodised } \\
\quad(\geq 15 \mathrm{ppm})\end{array}$}} & \multicolumn{4}{|c|}{ Adequately iodised salt categorized } \\
\hline & & & & & & & & \multicolumn{2}{|c|}{ 15-29.9 ppm } & \multicolumn{2}{|c|}{$\geq 30 \mathrm{ppm}$} \\
\hline & & $n$ & $\%$ & $n$ & $\%$ & $n$ & $\%$ & $n$ & $\%$ & $n$ & $\%$ \\
\hline \multicolumn{12}{|l|}{ Use of packaged salt } \\
\hline Packaged & 5095 & 337 & $6 \cdot 6$ & 680 & $13 \cdot 3$ & 4078 & $80 \cdot 0$ & 2137 & 41.9 & 1941 & $38 \cdot 1$ \\
\hline Not packaged & 587 & 132 & 22.5 & 131 & $22 \cdot 3$ & 324 & $55 \cdot 2$ & 197 & 33.6 & 127 & $21 \cdot 6$ \\
\hline \multicolumn{12}{|l|}{ Type of salt used } \\
\hline Refined & 3784 & 48 & 1.3 & 354 & $9 \cdot 4$ & 3382 & 89.4 & 1587 & 41.9 & 1795 & $47 \cdot 4$ \\
\hline Washed & 1321 & 138 & $10 \cdot 4$ & 314 & 23.8 & 869 & $65 \cdot 8$ & 649 & $49 \cdot 1$ & 220 & $16 \cdot 7$ \\
\hline Crystal & 414 & 237 & $57 \cdot 2$ & 83 & $20 \cdot 0$ & 94 & $22 \cdot 7$ & 58 & $14 \cdot 0$ & 36 & 8.7 \\
\hline 'Phoda' & 128 & 36 & $28 \cdot 1$ & 55 & $43 \cdot 0$ & 37 & 28.9 & 31 & $24 \cdot 2$ & 6 & 4.7 \\
\hline \multicolumn{12}{|l|}{ Multidimensional Poverty Index (MPI) } \\
\hline Not deprived (MPI score < 1/3) & 4262 & 330 & $7 \cdot 7$ & 525 & $12 \cdot 3$ & 3407 & $79 \cdot 9$ & 1720 & $40 \cdot 4$ & 1687 & 39.6 \\
\hline Deprived (MPI score $\geq 1 / 3$ ) & 1414 & 135 & 9.5 & 286 & $20 \cdot 2$ & 993 & $70 \cdot 2$ & 613 & 43.4 & 380 & $26 \cdot 9$ \\
\hline \multicolumn{12}{|l|}{ Type of diet } \\
\hline Not diverse diet & 3791 & 350 & $9 \cdot 2$ & 594 & $15 \cdot 7$ & 2847 & $75 \cdot 1$ & 1558 & $41 \cdot 1$ & 1289 & $34 \cdot 0$ \\
\hline Diverse diet & 1891 & 119 & $6 \cdot 3$ & 217 & 11.5 & 1555 & $82 \cdot 2$ & 776 & 41.0 & 779 & 41.2 \\
\hline \multicolumn{12}{|l|}{ Household food security $\dagger$} \\
\hline Secure & 4138 & 345 & $8 \cdot 3$ & 571 & $13 \cdot 8$ & 3222 & $77 \cdot 9$ & 1665 & $40 \cdot 2$ & 1557 & $37 \cdot 6$ \\
\hline Mildly secure & 497 & 53 & $10 \cdot 7$ & 63 & $12 \cdot 7$ & 381 & $76 \cdot 7$ & 184 & $37 \cdot 0$ & 197 & $39 \cdot 6$ \\
\hline Moderately insecure & 290 & 19 & $6 \cdot 6$ & 50 & $17 \cdot 2$ & 221 & $76 \cdot 2$ & 133 & $45 \cdot 9$ & 88 & $30 \cdot 3$ \\
\hline Severely insecure & 757 & 52 & $6 \cdot 9$ & 127 & $16 \cdot 8$ & 578 & $76 \cdot 4$ & 352 & $46 \cdot 5$ & 226 & 29.9 \\
\hline
\end{tabular}

*Percentages represent row-wise percentages.

†Basic information on whether a household is food secure or not is a component of the MPI score; however, it is useful to also see the association of different levels of food security with access to adequately iodised salt separately.

and in households using refined salt. However, the encouraging national-level progress in iodised salt coverage in India masks significant differentials by rural/urban, zonal and socio-economic indicators. The low iodised salt coverage in rural areas compared with urban areas has serious implications for the development potential of children belonging to rural populations. The key interventions identified to reach these marginalised populations are enhanced monitoring of the edible salt supply chain in these areas, inclusion of iodised salt in the public distribution system (PDS) and other social safety programmes, and special schemes to provide good-quality iodised salt in these high-burden areas.

Wide variations in iodised salt coverage are seen across the six zones in the survey, with a difference in the range of 25 percentage points between coverage in the South zone compared with the North zone. The North zone also shows higher than national coverage of iodised salt, whereas Central, East and West zones show coverage similar to the national level. The South zone, primarily because of the higher percentage use of non-refined (crystal) salt than any other zone, continues to be way below the national average of iodised salt coverage. The complex supply chain dynamics of salt is an important factor influencing availability of adequately iodised salt in different geographical areas. The survey results show that coverage continues to be high in the North-East zone, where edible salt is transported predominantly by rail and which has a nominee system (only a few licensed traders are permitted to import salt in the state). Both the above factors ensure high coverage of iodised salt by ensuring optimal regulation of the quality salt iodisation. In India transport of iodised salt by rail requires certification by the Salt Commissioner's Office, thus ensuring better production and monitoring of iodine content ${ }^{(25)}$. No such certification is required for salt transported by road.

The level of iodisation is higher in the refined and packaged salt category compared with the non-refined and non-packaged salt category. Under the NIDDCP and the Food Safety and Standards Authority of India, there is a ban on the sale of non-iodised salt in India ${ }^{(26)}$. In recent years there has been increased advocacy for more stringent implementation of the provision of the law pertaining to the ban on non-iodised salt. In addition, an attempt has also been made with production-end interventions to nudge salt manufacturers away from unrefined and unpackaged salt production to refined and packaged salt. Assey et al. have shown that knap-sack spraying and manual mixing yielded much more homogeneous iodisation of salt in salt bags at small-scale salt producers ${ }^{(27)}$. Several projects have been undertaken to supply packaging material to small- and medium-scale producers, to support them for technology upgrading and setting up of refineries ${ }^{(28)}$. Also, the NIDDCP should focus on households consuming non-refined and non-packaged salt and ensure adequate iodisation of the salt consumed by them. It is technically feasible to adequately iodise non-refined washed and crystal salt, and the regulatory authorities 
Table 4 Association of selected factors with adequately iodised salt consumption in India; first National lodine and Salt Intake (NISI) Survey 2014-2015

\begin{tabular}{|c|c|c|c|c|c|c|c|c|}
\hline \multirow[b]{2}{*}{ Characteristic } & \multicolumn{2}{|c|}{$\begin{array}{c}\text { Adequately iodised salt } \\
(\geq 15 \mathrm{ppm}) \\
(n 4402) \\
\end{array}$} & \multicolumn{2}{|c|}{$\begin{array}{c}\text { Inadequately iodised salt } \\
(<15 \mathrm{ppm}) \\
(n 1280) \\
\end{array}$} & \multirow{2}{*}{$\begin{array}{l}\text { Unadjusted } \\
\text { OR }\end{array}$} & \multirow{2}{*}{\multicolumn{2}{|c|}{$\begin{array}{lc} & \text { Adjusted } \\
95 \% \mathrm{Cl} & \text { OR }\end{array}$}} & \multirow[b]{2}{*}{$95 \% \mathrm{Cl}$} \\
\hline & $n$ & $\%$ & $n$ & $\%$ & & & & \\
\hline \multicolumn{9}{|l|}{ Type of residence } \\
\hline Rural & 1964 & 44.6 & 880 & $68 \cdot 8$ & 0.37 & $0.32,0.42$ & 0.55 & $0.47,0.64$ \\
\hline Urban & 2438 & 55.4 & 400 & 31.3 & 1.00 & Ref. & 1.00 & Ref. \\
\hline \multicolumn{9}{|l|}{ Education } \\
\hline Illiterate & 1103 & $25 \cdot 1$ & 426 & 33.3 & 0.67 & $0.59,0.77$ & $1 \cdot 13$ & $0.96,1.32$ \\
\hline Literate & 3299 & 74.9 & 854 & $66 \cdot 7$ & 1.00 & Ref. & 1.00 & Ref. \\
\hline \multicolumn{9}{|l|}{ Caste } \\
\hline SC/ST/backward/lower & 2795 & $64 \cdot 1$ & 956 & $74 \cdot 8$ & 0.60 & $0.52,0.69$ & 0.84 & $0.72,0.98$ \\
\hline Others & 1566 & 35.9 & 322 & $25 \cdot 2$ & 1.00 & Ref. & 1.00 & Ref. \\
\hline \multicolumn{9}{|l|}{ Multidimensional Poverty Index (MPI) } \\
\hline Deprived (MPI score $\geq 1 / 3$ ) & 993 & $22 \cdot 6$ & 421 & 33.0 & 0.59 & $0.52,0.68$ & 0.72 & $0.61,0.85$ \\
\hline Not deprived (MPI score $<1 / 3$ ) & 3407 & 77.4 & 855 & $67 \cdot 0$ & 1.00 & Ref. & 1.00 & Ref. \\
\hline \multicolumn{9}{|l|}{ Type of diet } \\
\hline Non-diverse diet & 2847 & 64.7 & 944 & 73.8 & 0.65 & $0.57,0.80$ & 0.73 & $0.62,0.86$ \\
\hline Diverse diet & 1555 & $35 \cdot 3$ & 336 & $26 \cdot 3$ & 1.00 & Ref. & 1.00 & Ref. \\
\hline \multicolumn{9}{|l|}{ Use of packaged salt } \\
\hline Not packaged & 324 & 7.4 & 263 & 20.5 & 0.31 & $0.26,0.38$ & 0.48 & $0.39,0.59$ \\
\hline Packaged & 4078 & 92.6 & 1017 & 79.5 & 1.00 & Ref. & 1.00 & Ref. \\
\hline \multicolumn{9}{|l|}{ Use of refined salt } \\
\hline Not refined & 1020 & $23 \cdot 2$ & 878 & $68 \cdot 6$ & 0.14 & $0 \cdot 12,0 \cdot 16$ & 0.17 & $0.15,0.20$ \\
\hline Refined & 3382 & $76 \cdot 8$ & 402 & 31.4 & 1.00 & Ref. & 1.00 & Ref. \\
\hline
\end{tabular}

SC, scheduled caste; ST, scheduled tribe; Ref., reference category.

should strictly ensure compliance of the same by the nonrefined edible salt producers. 'Phoda' salt (large granules) is still consumed in a few selected pockets; it should be phased out and replaced with refined salt as it is not feasible to iodise 'phoda' salt.

The present survey is the first national-level survey of iodised salt coverage which used the iodometric titration method. All earlier estimates of iodised salt coverage in India were done using STK, which are relatively less reliable. As part of the current survey, STK were also used to estimate the salt iodine content and STK results were compared with iodometric titration to assess the validity of STK. The observed sensitivity of STK at cut-off of $0 \mathrm{ppm}$ was $95.9 \%$ but specificity was only $44.6 \%$. The sensitivity and specificity at the cut-off of $15 \mathrm{ppm}$ were 84.5 and $49.2 \%$, respectively. Thus, overall, STK are not a valid and accurate method to ascertain the iodine content of salt as reflected by low sensitivity and specificity.

Use of the MPI helped in assessing household vulnerability to poverty, a key factor affecting iodised salt coverage. The high differential coverage of adequately iodised salt in deprived and non-deprived households, seen in the present survey, warrants identification of strategies to reach out to deprived households to bridge this gap. The PDS, with its massive outreach to populations who are below poverty line and vulnerable across India, provides an ideal platform to reach the unreached. The same may be leveraged to ensure adequately iodised salt consumption by deprived households. However, currently only $5 \%$ of households procure edible salt from the PDS and edible salt is not listed as an essential commodity for the PDS in the country. There have been a few successful models of supply of iodised salt in India, including Tamil Nadu, Chhattisgarh and Bihar. There is need to scale up these successful models of providing lowcost, good-quality adequately iodised salt in the essential commodity basket of the PDS in the country.

One of the strengths of the present survey is high response rate (specially for the salt samples), ensuring the representativeness of the survey findings. External field survey validation by public health professionals independently of survey teams ensured quality data collection. Robust internal and external quality assurance methods were followed for the laboratory analysis of salt samples.

The survey limitations include non-availability of statelevel disaggregated information. However, this was decided at the design stage itself and was due primarily to paucity of resources as well as the fact that the edible salt supply chain may be affected at a more proximal point than state level. Accordingly, six geographical regions were selected, factoring in aggregations of states likely to share the same characteristics as far as the supply chain of edible salt is concerned. The survey did not include the retailers and wholesalers, who may have provided additional valuable information. Also, keeping in view the increase in consumption of processed food, it is important 
that future iodised salt coverage surveys also take account of use of iodised salt in processed food in addition to estimating household iodised salt use.

The findings of the survey helped in identifying the following key programme recommendations to attain USI in India: strengthen implementation of the regulations for mandatory iodisation of salt set out in the Food Safety and Standards Act of India ${ }^{(29)}$; universalise iodised salt in the PDS and include iodised salt in the essential commodity basket of the PDS; modernise the salt industry including technology for the iodisation of non-refined salt; and strengthen advocacy on the role of iodine intake in brain development and its linkage to learning abilities, school education and national development. Implementation of these recommendations will lead to increased overall access to adequately iodised salt and minimise the differentials in household coverage of adequately iodised salt observed between zones, rural/urban and socio-economic levels.

The NISI 2014-2015, the first national (and subnational) representative survey in India, while documenting the public health success story of USI in India, also provides evidence for significant differentials in coverage across zonal, rural/urban and socio-economic (high MPI/ low MPI) strata. The valuable evidence generated by the survey should provide inputs to sustain, accelerate and achieve USI and IDD elimination in India.

\section{Acknowledgements}

Acknowledgements: The authors thank Dr Jacky Knowles (Global Alliance for Improved Nutrition (GAIN)), Dr Jonathan Gorstein (Iodine Global Network), Dr Rajan Sankar (GAIN), Dr Jee Hyun Rah (UNICEF) and Dr Pradeep Saxena (Advisor (Nutrition), Ministry of Health and Family Welfare, Government of India). Financial support: This study was funded by GAIN-UNICEF USI Partnership Project. The funding organisation had no role in the design/conduct of the study, collection/analysis/ interpretation of the data or preparation/review/approval of the manuscript. Conflict of interest: None. Authorship: C.S.P. and K.Y. were involved in formulating the research question; C.S.P., K.Y., H.R.S., R.K. and A.C. designed and carried out the study; C.S.P., K.Y., H.R.S. and A.D.G. were involved in analysing the data and writing the article. Ethics of buman subject participation: This study was conducted according to the guidelines laid down in the Declaration of Helsinki and all procedures involving human subjects were approved by the Institutes Ethical Committee of the All India Institute of Medical Sciences, New Delhi. Informed written consent was taken from the literate participants of the survey. For illiterate respondents, the consent form was read aloud in the presence of a literate witness and verbal consent of the respondent duly signed by the witness was obtained.

\section{Supplementary material}

To view supplementary material for this article, please visit https://doi.org/10.1017/S1368980018002306

\section{References}

1. Andersson M, Karumbunathan V \& Zimmermann MB (2012) Global iodine status in 2011 and trends over the past decade. J Nutr 142, 744-750.

2. Kochupillai N (2017) Neonatal hypothyroidism in India. $M t$ Sinai J Med 59, 111-115.

3. Kumar S \& Berkman LF (2015) Association of inadequately iodized salt use with underweight among young children in India. Asia Pac J Public Health 27, 185-194.

4. Krämer M, Kupka R, Subramanian SV et al. (2016) Association between household unavailability of iodized salt and child growth: evidence from 89 demographic and health surveys. Am J Clin Nutr 104, 1093-1100.

5. Hynes KL, Otahal P, Hay I et al. (2013) Mild iodine deficiency during pregnancy is associated with reduced educational outcomes in the offspring: 9-year follow-up of the gestational iodine cohort. J Clin Endocrinol Metab 98, 1954-1962.

6. Bleichrodt N \& Born MP (1996) A metaanalysis of research on iodine and its relationship to cognitive development. In The Damaged Brain of Iodine Deficiency, pp. 195-200 [JB Stanbury, editor]. New York: Cognizant Communication Corporation.

7. Bath SC, Steer CD, Golding J et al. (2013) Effect of inadequate iodine status in UK pregnant women on cognitive outcomes in their children: results from the Avon Longitudinal Study of Parents and Children (ALSPAC). Lancet 382, 331-337.

8. Pandav CS (2006) Tracking Progress Towards Sustaining Elimination of IDD in Seven States 1999-2005. http://www. iqplusin.org/index.php/publications-reports/other-technicalreports (accessed December 2016).

9. UNICEF-World Health Organization Joint Committee on Health Policy Special Session (1994) World Summit for Children - Mid-Decade Goal: Iodine Deficiency Disorders (IDD). http://www.ign.org/document.cfm?page_id=142003337 (accessed August 2018).

10. Aburto NJ, Abudou M, Candeias V et al. (2014) Effect of salt iodization to prevent iodine deficiency disorders: a systematic review with meta-analyses. http://apps.who.int/iris/ bitstream/handle/10665/148175/9789241508285_eng.pdf? sequence $=1$ (accessed August 2018).

11. Sooch SS, Deo MG, Karmarkar MG et al. (1973) Prevention of endemic goitre with iodized salt. Bull World Health Organ 49, 307-312.

12. Ministry of Health and Family Welfare, Government of India (2006) Revised Policy Guidelines on National Iodine Deficiency Disorders Control Programme. http://pbhealth.gov.in/ Revised\%20Policy\%20Guidelines\%20Govt.\%20of\%20India.pdf (accessed July 2017).

13. Ministry of Health and Family Welfare, Government of India \& International Institute of Population Sciences (2007) National Family Health Survey-3 National Report (2005-06). http://rchiips.org/nfhs/nfhs3_national_report.shtml (accessed July 2017).

14. UNICEF India Country Office (2009) National Factsheet: Coverage Evaluation Survey 2009. http://hshrc.gov.in/wpcontent/uploads/National_Fact_Sheet_CES_2009.pdf (accessed June 2017)

15. Ministry of Health and Family Welfare, Government of India \& International Institute of Population Sciences (2017) National Family Health Survey-4, 2015-16: India Fact Sheet. 
http://rchiips.org/NFHS/pdf/NFHS4/India.pdf (accessed March 2017).

16. Pandav CS, Arora NK, Krishnan A et al. (2000) Validation of spot-testing kits to determine iodine content in salt. Bull World Health Organ 78, 975-980.

17. World Health Organization (2010) Sustaining the elimination of iodine deficiency disorders (resolution WHA60.21). In Sixty-Third World Health Assembly, Provisional Agenda Item 11.24, Progress Reports. http://www.who.int/nutrition/ topics/A63.27_idd_en.pdf?ua=1 (accessed July 2017).

18. Inter-State Council Secretariat, Ministry of Home Affairs, Government of India (2017) Zonal Councils I Genesis. http:// interstatecouncil.nic.in/genesis/ (accessed June 2013).

19. International Council for Control of Iodine Deficiency Disorders, UNICEF \& World Health Organization (2001) Methodology for selection of survey sites by PPS sampling (Annex 4). In Assessment of Iodine Deficiency Disorders and Monitoring their Elimination: A Guide for Programme Managers, 2nd ed., pp. 77-84. http://www.ign.org/ cm_data/2001_IDD_Assessment_manual_idd_01_WHO.pdf (accessed July 2017).

20. Alkire S, Conconi A \& Roche JM (2013) Multidimensional Poverty Index 2013: Brief Methodological Note and Results. http://www.ophi.org.uk/wp-content/uploads/MPI-2013-BriefMethodological-Note-and-Results1.pdf (accessed June 2017).

21. Coates J, Swindale A \& Bilinsky P (2007) Household Food Insecurity Access Scale (HFIAS) for Measurement of Food Access: Indicator Guide. Version 3. https://www.fantapro ject.org/sites/default/files/resources/HFIAS_ENG_v3_Aug 07.pdf (accessed June 2013).

22. Santos ME \& Alkire S (2011) Training Material for Producing National Human Development Reports. http://hdr.undp.org/ sites/default/files/mpi_trainingmaterial_mcc_mk_clean_june_ 2015.pdf (accessed November 2017).
23. National Sample Survey Office, Ministry of Statistics and Programme Implementation, Government of India (2014) NSSO Report No. 558 (68/1.0/2). Household Consumption of Various Goods and Services in India 2011-12. NSS 68th Round (July 2011-June 2012). http://mospi.nic.in/sites/ default/files/publication_reports/Report_no558_rou68_30 june14.pdf (accessed July 2018).

24. Borkotoky K, Unisa S \& Gupta AK (2018) State-level dietary diversity as a contextual determinant of nutritional status of children in India: a multilevel approach. J Biosoc Sci 50, $26-52$.

25. Salt Department, Government of India, Ministry of Commerce and Industry, Department of Industrial Policy and Promotion (2017) Annual Report 2015-2016. http://www. saltcomindia.gov.in/salt-ar-2016.pdf (accessed July 2017).

26. Pandav CS (2013) Evolution of iodine deficiency disorders control program in India: a journey of 5,000 years. Indian J Public Health 57, 126-132.

27. Assey VD, Tylleskär T, Momburi PB et al. (2009) Improved salt iodation methods for small-scale salt producers in lowresource settings in Tanzania. BMC Public Health 9, 187.

28. Salt Department, Government of India, Ministry of Commerce and Industry, Department of Industrial Policy and Promotion (2015) Annual Report 2014-2015. http://salt comindia.gov.in/salt-ar-2015a.pdf (accessed July 2018).

29. Ministry of Health and Family Welfare (Food Safety and Standards Authority of India), Government of India (2011) Food Safety and Standards (Food Products Standards and Food Additives) Regulations, 2011. Gazette of India: Extraordinary, Part III-Section 4, 287-448. http://www.old. fssai.gov.in/Portals/0/Pdf/Food\%20safety\%20and\%20stan dards $\% 20$ (Food $\% 20$ product $\% 20$ standards $\% 20$ and $\% 20$ Food $\%$ 20Additives)\%20regulation,\%202011.pdf (accessed August 2018). 